\section{a Pectures}

ON THE

\section{DIAGNOSIS AND TREATMENT OF DISEASES OF WOMEN.}

DFLIVERED AT ST. MAHY'S HOSPITAL MEDICAL SCHOOL.

BY

GRAILY HEWITT, M.1.LoNd., M.R.C.P.,

PHYSICIAN TO THE BRITISH LYING-IN HOSPITAL; LECTURER ON MHDWIFERY AND DISEASES OF WOMEN AND CHILDREN AT ST. MARY'S HOSPITAL MEDICAL SCHOOL.

Disorders of Menstruation (continued).

Minus Conditions of Catamenia: Treatinent. Gentuemen, - The treatment of cases coming under the categories now severally considered is a very important subject. It has been seen how very many different causes may give rise to "absence of menstruation". In many such cases, to have arrived at a complete diagnosis is to know what the treatment should be. Hence the extreme utility of pushing the diagnosis as far as possible. The treatment of cases of "amenorrhœa", as they are called, is generally of a very empirical character; the great object is to bring on the secretion; the real cause of the absence of menstruation being overlooked, or not looked after. Cases in which absence of menstruation is due to organic disease of the generative organs, for instance, have been frequently treated by administration of emmenagogues.

The treatment of cases where retention is present will be discussed in another place. In cases where the uterus is absent, the question of treatment does not require much consideration. Practically, the first question we have to discuss, as regards treatment, is the management of those cases in which puberty is delayed, and this is the cause of the amenorrhœa.

Now, as long as the health of the patient appears good, the proper treatment may be to let matters take their course, and to wait patiently, the more especially if there be no menstrual molimina. In cases where the absence of menstruation is connected with the presence of an undersized uterusi.e., where the uterus is imperfectly developed-Dr. Simpson recommends the continued wearing of a series of small galvanic pessaries of greater and greater length and thickness. The irritation which the presence of the foreign body in question produces, leads, it is stated, to the gradual development of the organ, and to the occurrence of menstruation. Cases in which the uterus is only a few lines shorter than the normal uterus are those only to which this method of treatment is stated to be applicable. (Med. Times and Gaz., June 15th, 1861.) Here I would only remark, that it must be a matter of great difficulty to substantiate the effect of these remedial measures in inducing the gradual development of the organ here spoken of : the mere production of a sanguineous discharge from the uterus cannot be held to be a sufficient evidence of it.

The second and most important question is the treatment of those cases where the absence of menstruation and disorder of the general health are associated. Notwithstanding the fact that, in the writings of all recent good authorities, we find a con tinued protest against the plan of attempting in these cases to restore or produce the menstrual secretion by direct means, there is still a vast amount of prejudice and ignorance on this subject to be dispelled even in professional circles. We still hear emmenagogues spoken of, and particular medicines or remedies extolled for their efficacy in this respect, as if the restoration or production of the secretion were the only object in view. It is not that the teaching on this subject has been bad, but that the teaching has been so little attended to.

It is impossible to put the matter better or more forcibly than in the words of Sir Charles M. Clarke, which are as follows:-

"The function of menstruation, like the other functions of the body, is best performed when the system is in health. Now, health is not constituted by excess of fulness, or by the performance of violent actions, any more than by debility or enfeebled action; consequently, the exhibition of stimulants will not influence this secretion, unless attention be given to the restoration of the general health of the patient, even in cases of debility. Still less will such a mode of treatment be applicable to cases of interrupted menstruation occurring in plethoric habits, where the presence of the plethora itself is the cause of the interruption of the due performance of the natural secretions. Instead, then, of resorting to such measures, to the employment of the whip and of the spur in such cases (when, if they do anything, they do mischief), let the morbid peculiarities of the constitution and the habits of life of the patient be taken into consideration; let the first be counteracted, the second be improved; let the sanguine have her excess of fulness diminished, let the debilitated have her powers augmented: in short, let the general health be amended, and the functions of health will be restored." (Diseases of Females, Part II, p. 38.$)$

In the works of Marshall Hall, Rigby, and other equally eminent, writers, we find the same doctrines inculcated, and the fruitlessness and absurdity of attempting by so-called emmenagogues alone to cure amenorrhœa coexisting with impaired health, pointed out. It must be held to be decidedly improper by local stimulation of the uterus, as by continued use of metallic pessaries or otherwise, to attempt to produce a menstrual flow in a phthisical patient for instance, certainly to give a prominent place to such treatment; indeed, to practitioners imbued with the principles of the eminent men above alluded to, such practice must appear something worse than absurd. It is the experience of all observant practitioners that those remedies act most efficiently as enmenagogues which produce a most decidedly beneficial effect on the defective condition of the general health. In treating such cases successfully, the menstrual secretion is the final step to be reached. Improvement in other respects must be effected first; the rest will follow as a matter of course, in the vast majority of cases.

The treatment, then, must be general,-to find out what is the weak point, and to attack this. Either the patient has been living badly, taking too little food, or food not sufficiently nutritious; or she 
has been leading a too sedentary or artificial life, deprived of pure air-in short, subjecting the body at a very critical period to many influences known to be incompatible with sound health. Medicines are quite subordinate in importance to the removal of these defective hygienic conditions.

No rule applicable in all cases can be laid down; and in different cases very opposite methods of treatment may have to be insisted upon. We generally do find, however, as an effect of the bad state of health of the patient, partly also as a cause of the same, that there is great sluggishness and inactivity of the digestive organs, evinced by want of appetite and constipation; and hence, before it is possible to administer the amount of nutritious food the patient requires, it is frequently necessary to effect an improvement in the condition of the digestive organs.

The dyspepsia often present in such cases is a most troublesome complication; and is best treated by administering, frequently and in very small quantities, for some days together, food of the simplest character; avoiding all solid matters, and giving the patient only such food as it may be found by experiment she is able to digest freely and easily. Milk and water, weak beef-tea, yelk of egg beaten up uncooked, with milk, these are some of the most nutritious and easily digested foods.

The sluggish, inactive state of the digestive organs will be improved by administering a dose of blue pill and compound colocynth pill every night for two or three nights, followed the next morning by a draught containing Rochelle salt, or sulphate of potash, with manna and rhubarb, as recommended by Dr. Rigby. Hygienic measures, exercise in the open air, cold sponging, friction with a rough towel, warm clothing, etc., to be associated then and subsequently. After pursuance of this treatment for some days, tonics, as iron and quinine, to be given twice or thrice daily, a laxative every morning, and twice a week the blue pill and colocynth.

I believe that the same good effects may be obtained, and with less discomfort to the patient, by administering one good purge at the first, and by subsequently giving a small dose (half an ounce) of the compound iron mixture thrice a day; care being taken to procure action of the bowels by giving every other morning confection of senna, castor-oil, or an effervescing draught containing Rochelle salt. The small dose of iron appears to act quite as efficiently as the larger one. A preparation of iron less nauseous than the compound mixture has been recommended; namely, the syrup of the perphosphate of iron.

Again, I would say, bathing, frictions of the skin, exercise out of doors, in short, every meaus that can be devised to put the body in a sound state of health, will be beneficial as regards the end in view, the induction of menstruation. This point must ever be kept in view: amenorrhœe is only a symptom, not a disease.

Are emmenagogues, then, never to be given with the view of producing in a more direct and immediate manner the catamenial flow? But rarely. They are more especially applicable in the cases to be presently considered where there is suppression, and where the menses have been present. The actual and immediate production of the menstrual flow in the class of cases now concerned is, however, advantageous in one way, that it sets at rest any doubt we may have as to the possibility of menstruation. And the more direct action may be sought to be induced in cases where general measures have been fairly tried and found unavailing; also, in cases where the general health being good, and no attempt at menstruation observed, it is thought expedient to try this method of treatment as a kind of dernier ressort.

The best method to follow in endeavouring to induce directly this action of the uterus will be considered presently.

Treatment of Cases of Menstrual Suppression. If the discharge have been observed once or twice, and then to a slight extent, and have then ceased to appear, the patient being in a bad state of health, the case is practically identical with those just considered, and must be treated on the same principles; the health is to be improved by every available means, and the discharge will then return.

The next class of cases are those in which the discharge has been well established and has been suddenly suppressed.

In a case of acute suppression of the menses, if seen in time, the proper treatment would be to place the patient immediately in a warm hip-bath, and to administer a stimulant, such as hot gin and water, and, especially if a sudden chill be the cause, to endeavour to excite the action of the skin, by giving a dose of Dover's powder. The application of a sinapism to the hypogastric region, the application of leeches to the vulva, have been also recommended. It is probable that the most powerful means of inducing the return of the discharge under such circumstances would be either the application of electro-galvanism, or the administration of an enema containing aloes by the rectum. It is generally the case, however, that when the patient is seen, the period for such treatment is gone by. We must then wait uritil about the time of the next period, and then apply the remedies in question.

Many different medicines or remedial measures are set down as efficacious in inducing the flow of the menses, and which are given or directed to be given with the view of exciting, and in a direct manner, the menstrual secretion. It is to be remarked of these, that they are exceedingly uncertain in their effects and action in different individuals, and very frequently have no effect whatever. Moreover, it does not follow, as I have already remarked, that because a discharge has been procured the difficulty is forthwith at an end ; the simple production of a sanguineous discharge is not the only object we have in view. It is not so difficult a thing to produce a discharge, as it is to reproduce what may be considered to constitute the discharge of menstruation.

To attempt even an enumeration of the remedies which have been used or recommended for the purpose of restoring or producing the menstrual flow would be a profitless task. Most of them act, it must be concluded, by producing congestion, and fulness of the vessels of the uterus and surrounding parts. The following are some most recommended of late years :-aloes, in form of enema ; liquor ammoniæ, dissolved in milk (a teaspoonful of the ammonia in a pint of milk injected into the vagina); savin (Sir Charles Clarke, Dr. Tilt, and others); iodine (Dr. Rigby, who preferred it in the form of iodide of iron) ; ergot of rye (Rigby). This list might be almost indefinitely extended. 
Dr. Simpson recommends the application of direct stimulants to the interior of the uterus-nitrate of silver, cantharides, or iodine-by means of a portecaustique, the application to be made at the time when menstruation should occur, and repeated at monthly intervals. The same authority speaks highly of a kind of dry-cupping of the interior of the uterus, and of the employment of galvanic intrauterine pessaries of peculiar construction in the form of amenorrhœea now under consideration. With respect to the efficacy of galvanism, Dr. Simpson considers its continued application by the use of pessaries more serviceable than the occasional application by the ordinary methods. Dr. Althaus states, however, that he has in many cases found great benefit from Faradisation assiduously and properly applied; and, in the very few cases in which such direct local treatment by galvanism or by other measures of an analogous kind is necessary, the latter method is to be recommended as the preferable one.

\section{fllurstrations}

OF

HOSPITAL PRACTICE: METROPOLITAN AND PROVINOLAL.

\section{LONDON HOSPITAL.}

INGUINO-SCROTAL TUMOUR OF RIGHT SIDE.

Under the care of G. Critchetr, Esq., and C. F. Maunder, Esq.

J. A., aged 32 , a healthy-looking countryman, had been ruptured twelve years, and worn a truss during the last seven. He said that, on August 25th, he was kicked by a horse upon the pad of the truss, and knocked down, when the rupture redescended, and could not be returned by himself. On admission (Aug. 28th), a pyriform tunnour, base downwards, occupied the internal half of the inguinal region and right side of the scrotum; it was firm, elastic, not translucent, painful just below the external abdominal ring; and corresponding with the position of the latter a slight bulging and dis. tinct impulse occurred on conghing. This impulse was communicated, but with much less intensity, to the whole swelling. The abdominal viscera performed their functions healthily. He was ordered to keep in bed, with an evaporating lotion to the tumour.

Sept. 1st. The tumour was much the same. In the absence of Mr. Critchett, and in order to determine the diagnosis, Mr. Maunder put the patient on a diet of bread and potatoes only, with two pints of fluid during the fourand.twenty hours, with a view to promote absorption of adventitious substances which might possibly conceal the nature of the swelling. The patient was ordered to take a dose of house-medicine every morning.

Sept. 3rd. The tumour was smaller, and was now divided into two portions by a depression about its middle, giving it the appearance of being composed of a hydrocele below and a hernia above. The testicle could not be felt. Mr. Critchett tapped the lower swelling, und evacuated several ounces of fluid, well charged with blood. The testicle, smaller than usual, could now be felt; and exteriding up from this gland was an elongated mass, which received an impulse on coughing. The component parts of the mass, enlarged, indurated, and knotied, could be passed in review by the finger and thumb (doubtless a varicocele).

Sept. 14th. The tunica vaginalis, having refilled, was tapped, and several ounces of straw-coloured fluid were evacuated. It was then washed out with a strong solution of iodine; and on the 19th the patient, being desirous of going home, was allowed to leave; but betore reab. sorption of the effusion, consequent on injection, had taken place.

Remarks. This case is interesting, firstly, because it shows how cautiously the opinion of the patient must be received, even though, as in this case, he be "quite sure" that the hernia descended into the scrotum at the time of the accident, when the evidence is in favour of its not having advanced lately beyond the external ring; secondly, "impulse given to the whole tumour" is in favour of hernia, especially when the state of the cord cannot be known; while, thirdly, the sudden appearance of a tense, elastic, not translucent swelling of the scrotum, concealing the testis, was in favour of an effusion of blood (hæmatocele) into the previously healthy tunica vaginalis, but which, on tapping, did not contain sufficient blood to support that opinion, more especially as the patient afterwards sdmitted that "perhaps the purse had been larger than natural for some time", and in all probability had contained a hydrocele previous to the accident.

\section{Gibininal Communiations.}

\section{ON CHRONIC SUPPURATION OF TENDINOUS SHEATHS, SIMULATING AFFECTIONS OF JOINT'S.}

\section{By Richard Barweld, F.R.C.S., Assistant-Surgeon to} Charing Cross Hospital.

Acute suppurations of tendinous sheaths are so well known to every surgeon, they are accompanied by so much pain and constitutional disturbance, the situation and boundaries of the disease are so clearly defined, that the diagnosis is perfectly easy. But a slow inflam. mation of these structures is much less distinguishable from a chronic disease of the neighbouring joint. In theory, the one malady should be perfectly different from the other; we ought to find the fluctuation and swelling in each case confined within certain bounds, and extending in each iustance in different directions; the patient's health should suffer very much in the one case, and not at all or but very little in the other; in fact, there should be well marked distinctions, which no novice could overlook, and the one disease should be by no chance mistakeable for the other. He who expects, however, to find the diagnostic symptoms of local atiections as clear and definite as they are described in systematic treatises, will be hugely disappointed when he comes to bed-side practice, and more especially when he has to do with chronic disease.

The parts most liable to be affected with suppuration in a tendinous sheath, are of course the neighbourhood of the wrist and ankle. The process is often extrenely slow, and may commence at once as a chronic disease; but it more usually begins in a more or less acute form from injury or other cause. The pain, lameness, teuderness, and swelling, are at first pretty severe and defined; but in a little time they all diminish, and be. come more diffused. The patient, however, coes not get thoroughly well; he may be able to use the limb a little, but some pain and quaggy swelling continue, which again slowly increase. This swelling has never, after the first acute or subacute stage of the disease, $a$ defined clearly marked boundary; it fluctuates more or less obscurely, and through a mass of thickened intil. trated tissue. After a time, the matter points, and may be evacuated by the knife, or may find its own way out; 